\title{
ИСПОЛЬЗОВАНИЕ МУЛЬТИПЛИКАТИВНОЙ МОДЕЛИ БИОКЛИМАТИЧЕСКОГО ПОКАЗАТЕЛЯ ДЛЯ ОПРЕДЕЛЕНИЯ ЛОКАЛЬНЫХ УСЛОВИЙ БЕЗОПАСНОСТИ РАБОТ НА ОТКРЫТОМ ВОЗДУХЕ
}

\author{
К. Ю. Суковатов, Н.Н. Безуглова \\ Институт водных и экологических проблем СО РАН, Россия \\ Алтайский государственный университет, Россия \\ Поступила в редакиию 15 января 2019 г.
}

\begin{abstract}
Аннотация: В настоящей работе выполнена оценка рисков обморожения групп, работающих на открытом воздухе в районах падения (РП) отделяющихся частей ракет-носителей, запущенных с космодрома Восточный, для отдельных временных интервалов зимнего сезона 2017-2018 годов. Представлено обоснование выбора мультипликативной модели биоклиматического показателя и критериального условия для значений функции желательности $D_{i} \geq 0,37$, при котором сохраняется на некотором уровне возможность работы на открытом воздухе без обморожений открытых участков тела. С использованием мультипликативной модели биоклиматического показателя жесткости погоды рассчитаны комбинации значений температуры воздуха и скорости ветра для определенных территорий, при которых выполняются условия жесткости погоды «удовлетворительные и лучше». Установлены конкретные интервалы времени, когда сохраняется на некотором уровне возможность работы на открытом воздухе без обморожений открытых участков тела. Полученные результаты могут быть использованы в оперативной работе, при планировании работ в суровых погодных условиях, а также для краткосрочного прогноза условий работы на открытом воздухе без риска получения травм от холода.
\end{abstract}

Ключевые слова: модели обобщенного биоклиматического показателя, жесткость погоды, риски обморожения, районы падения отделяющихся частей ракет-носителей, космодром Восточный.

\section{Using a Multiplicative Bioclimatic Indicator Model to Determine Local Safety Conditions for Work Outdoors}

\section{K. Yu. Sukovatov, N. N. Bezuglova}

\begin{abstract}
In the present work, the chilblain risks for groups operating in the open air in the areas of falling rocket carrier separable parts launched from the Vostochny cosmodrome for specific time intervals of the 2017-2018 winter season were evaluated. The substantiation of the choice of the multiplicative bioclimatic indicator model and the criterion condition for the values of the desirability function $D_{i} \geq 0.37$ is presented. The chosen criterion condition preserves the possibility of working outdoors without frostbite of open body parts. Using a multiplicative bioclimatic indicator model of weather severity, combinations of air temperature and wind speed were calculated for certain territories, under which the weather severity conditions are «satisfactory and better». Specific time intervals have been established, when the ability to work outdoors without frostbite of open body parts remains at a certain level. The results can be used in operational work, when planning work in harsh weather conditions, as well as for a short-term forecast of working conditions in the open air without the risk of injury from cold.
\end{abstract}

Key words: generalized bioclimatic indicator models, weather severity, chilblain risks, areas of falling of rocket carrier separable parts, Vostochny cosmodrome. 


\section{ВВЕДЕНИЕ}

Суровые погодные условия, которые усложняют нормальную жизнедеятельность и работу, свойственны для значительной части территории РФ. При осуществлении деятельности на открытом воздухе в условиях низких температур в районах Крайнего Севера или приравненных к нему для персонала существует потенциальный риск переохлаждения всего организма (гипотермия), обморожения открытых участков тела, органов дыхания. Большое значение в защите человека от переохлаждения приобретает активное, целенаправленное регулирование неблагоприятного воздействия холода на организм человека. При необходимости проведения работ вне отапливаемых помещений в холодный сезон важно обеспечить достаточную теплозащиту организма человека с помощью средств индивидуальной защиты (СИЗ), например, одежды. Поскольку возможности необходимой защиты человека от охлаждения в суровых климатических условиях с помощью одной лишь одежды ограничены, необходимо определять время пребывания на холоде в зависимости от различных метеоусловий, физической активности, теплоизоляции СИЗ [6].

Цель данной статьи - оценить риски обморожения групп, работающих в суровых погодных условиях в районах падения отделяющихся частей ракет-носителей, запущенных с космодрома Восточный. Анализ условий работы на холоде без риска обморожения выполнен для территорий РП 981, 983, 985 для отдельных временных интервалов зимнего сезона 2017-2018 годов.

Указанные территории расположены в труднодоступной местности, в сотнях километров от населенных пунктов. К местам падения отработанных частей вылетает специальная служба для сбора и утилизации оставшихся фрагментов. Первая ступень, как правило, падает недалеко от места старта, вторая во время падения разрушается на части, которые оказываются на земле на расстоянии до ста километров друг от друга. Поскольку РП 981, 983, 985 находятся на территориях, приравненных к Крайнему Северу, поиски фрагментов приходится вести в суровых погодных условиях. Поэтому анализ рисков обморожения для персонала, работающего на открытом воздухе на территориях РП, является актуальной задачей [1].

При принятии решений о работе в жестких погодных условиях необходимы сведения о риске обморожения персонала. Под оценкой рисков подразумевается выявление возникающих в процессе труда опасностей, определение их величины и значимости возникающих рисков $[2,4,10]$.

\section{МАТЕРИАЛЫ И МЕТОДЫ}

В мировой практике для оценки степени холодовой нагрузки при работе на открытом воздухе используют индексы холодового стресса: в РФ введен интегральный показатель условий охлаждения (обморожения) (ИПУОО) [4]. В настоящее время в США и Канаде используется индекс приведенной температуры (wind chill temperature index, WST) $[7,8,10]$.

В статье [6] показано, что при использовании индексов холодового стресса (в том числе ИПУОО и $\mathrm{WST}_{\mathrm{Nws}}$, для одних и тех же значений предикторов (температуры воздуха и скорости ветра) можно получить различные результаты оценки рисков охлаждения или обморожения.

В ВУНЦ ВВС «Военно-воздушная академия им. проф. Н. Е. Жуковского и Ю. А. Гагарина» разработан специализированный (обобщенный) биоклиматический показатель безопасности работ на открытом воздухе в суровых погодных условиях. Подробно методика расчета оптимального обобщенного показателя жесткости климата за конкретный месяц описана в [6]. Суть заключается в том, что для некоторого пункта (станции или узла регулярной сетки) создают выборку многолетних данных о среднемесячных значениях температуры воздуха (Ti) и скорости ветра (Vi).

Для каждой пары данных $T i$ и $V i$, где $i=1, \ldots, n$ ( $n$ - объем выборки), определяют используемые индексы жесткости погоды, с их помощью рассчитывают частные функции желательности. Специализированный (обобщенный) биоклиматический показатель безопасности работ на открытом воздухе в суровых погодных условиях определяют на основании полученных функций желательности. В зависимости от характера и условий задачи для значений функции желательности выбирают критериальное условие, на основании которого определяют количество дней с выбранными условиями жесткости погоды.

Специализированный (обобщенный) показатель получен на основе синтеза аналитических индексов ИПУОО, WCI, NWS для определения возможности работы на открытом воздухе в суровых погодных условиях без риска обморожения в данном районе. Для достижения этой цели создают регрессионные модели, отражающие зависимость средних значений желательности работы на открытом воздухе без обморожений от заданных значений метеорологических характеристик. 
Оценка регрессионных моделей с помощью коэффициента детерминации показала, чтобы получить заданный уровень желательности работы на открытом воздухе без обморожений для умеренно суровых климатических условий (например, север Европейской части России, где зимой сказывается смягчающее влияние морей) можно использовать линейную модель, для более жестких условий следует применять мультипликативную модель [3]:

$$
r=A t^{\alpha} v^{\beta},
$$

где $r=1-D-$ показатель жесткости погоды, характеризующий риск обморожения персонала при работе в суровых погодных условиях; $A$ - коэффициент, учитывающий размерность единиц измерений предикторов; $t=(-1) T$ - температурный фактор холода; $v$ - фактор ветрового режима; $\alpha, \beta-$ так называемые коэффициенты эластичности [3]. Коэффициент эластичности - это числовой показатель, раскрывающий процентное изменение одной переменной в результате однопроцентного изменения другой переменной.

Форма мультипликативной модели позволяет анализировать влияние метеорологических факторов на риск обморожения (показатель жесткости погоды $r$ ).

Коэффициенты эластичности $\alpha, \beta$ показывают, на сколько процентов изменится значение показателя $r$ жесткости погоды от своего среднего значения при изменении температурного или ветрового факторов на $1 \%$ от соответствующих средних значений. При условии $\alpha>0, \beta<1$ дополнительное увеличение на один процент одного из факторов (холодового или ветрового) приводит к усилению риска обморожения менее чем на один процент.

Мультипликативно-степенная форма (1) имеет характерные признаки. 1. Поскольку условия обморожения определяются двумя предикторами (холодовым и ветровым факторами), то ни один из них не должен быть равен нулю. В противном случае результат обращается в ноль, поэтому функция (1) определена при $t>0, v>0.2$. С ростом одного из предикторов при постоянном значении другого величина зависимой переменной (показателя $r$ ) растет [6], т.е. при усилении одного из факторов (холодового или ветрового) значение показателя обморожения не должно уменьшаться.

При построении модели (1) необходимо учитывать, что скорость ветра не может быть равной нулю. Предполагается, что минимальная скорость ветра равна средней скорости пешехода 1,3 м/с [9].
Кроме того, следует исключить случаи с положительной температурой воздуха (рассматривается фактор холода), а также значение $T=0$.

В данной статье используется критериальное условие для значений функции желательности $D_{i} \geq 0,37$. Исследования показали [3], что $D_{i} \geq 0,37$ соответствует условиям жесткости погоды «удовлетворительные и лучше». Это означает, что сохраняется возможность работы на открытом воздухе без обморожений открытых участков тела. Предполагается, что работающий персонал имеет защитную одежду, соответствующую особым требованиям защиты от холода и приложения физических нагрузок (многослойная одежда, где каждый слой служит специальным целям) [2].

Анализируемая территория находится в глубине Азиатского континента и приравнена к районам Крайнего Севера (абсолютные минимумы температуры воздуха опускаются до отметок ниже $-60^{\circ} \mathrm{C}$ ).

Поскольку территории РП 981, 983, 985 находятся в жестких климатических условиях, для оценки рисков охлаждения и обморожений персонала, работающего на открытом воздухе, использовали мультипликативную модель (1).

Показатель $r$ жесткости погоды может меняться при изменении $t$ или $v$ на $1 \%$ от соответствующих средних значений. В течение суток температура воздуха может колебаться на $15 \%$ и более только за счет суточного хода, поэтому для получения более информативных результатов использовали срочные данные по температуре воздуха и скорости ветра (8 сроков измерений в сутки). Метеорологические данные получены с использованием web-сайта http://rp5.

\section{ОБСУЖДЕНИЕ РЕЗУЛЬТАТОВ}

Для оценки риска обморожения для конкретных временных интервалов (22-28.11.2017 для территории РП 981 и 9-28.11.2017 для территорий РП $983,985)$ была рассчитана величина $D_{i}$ по факти-

Таблииа

Значения температуры воздуха и скорости ветра, при которых выполняется условие $D_{i} \geq 0,37$

\begin{tabular}{|c|c|}
\hline $\begin{array}{c}\text { Скорость } \\
\text { ветра, } \mathrm{м} / \mathrm{c}\end{array}$ & $\begin{array}{c}\text { Температура } \\
\text { воздуха, }{ }^{\circ} \mathrm{C}\end{array}$ \\
\hline 1,3 & $-23,4$ \\
\hline 2,0 & $-21,1$ \\
\hline 3,0 & $-19,3$ \\
\hline 4,0 & $-18,0$ \\
\hline 5,0 & $-16,8$ \\
\hline
\end{tabular}




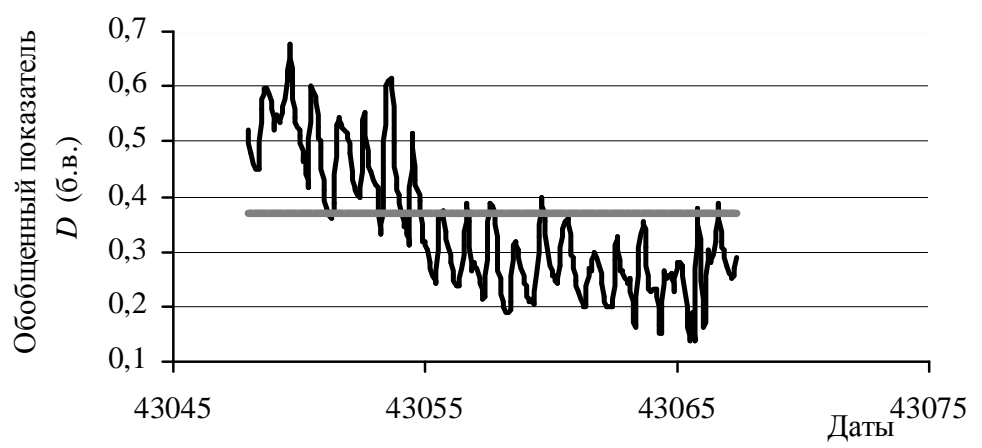

Puc. 1. Изменение обобщенного показателя $D_{i}$ за период 22-28.11.2017 для территории РП 981. Серая линия соответствует выбранному критическому значению обобщенного показателя $D=0,37$

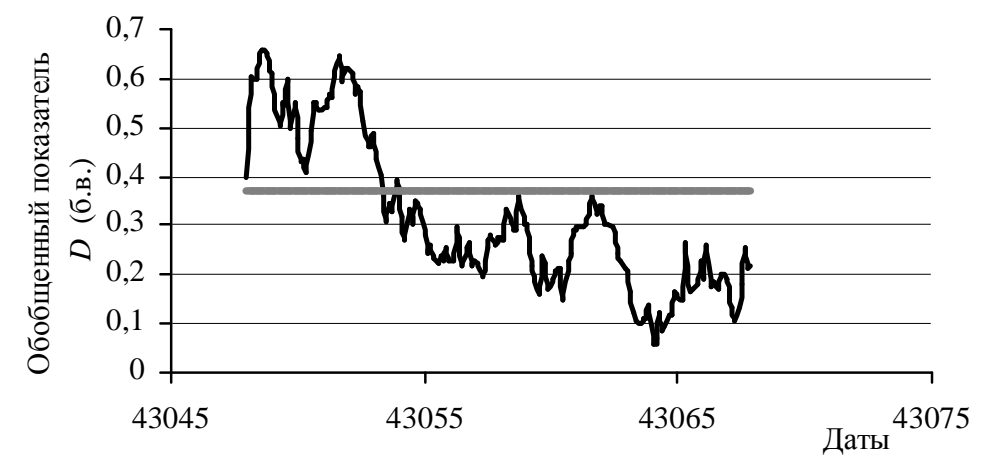

Puc. 2. Изменение обобщенного показателя $D_{i}$ за период 9-28.11.2017 для территории РП 983. Серая линия соответствует выбранному критическому значению обобщенного показателя $D=0,37$

ческим ежедневным данным, определены комбинации параметров $t_{i}$ и $v_{i}$, для которых выполняется условие $D_{i} \geq 0,37$.

В таблице представлены сочетания значений температуры воздуха и скорости ветра, при которых выполняется условие $D_{i} \geq 0,37$ - условия жесткости погоды «удовлетворительные и лучше». Это означает, что сохраняется возможность работы на открытом воздухе без обморожений открытых участков тела.

На рисунках 1-3 представлен временной ход параметра $D$ за анализируемые временные интервалы для территории каждого РП. Серая линия соответствует выбранному критическому значению обобщенного показателя $D=0,37$, область выше серой линии представляет собой временные интервалы, когда выполняются условия $D_{i} \geq 0,37$.

В течение анализируемого периода температура воздуха менялась от $-22,4$ до $-37,1^{\circ} \mathrm{C}$, средняя скорость ветра от 0 до 5 м/с. Как видно из графика, при работе на открытом воздухе даже при наличии соответствующей экипировки риск обморожений открытых участков тела присутствовал в течение всего временного интервала 22-28.11.2017. Исключение составил один срок: 27.11.2017 15.00, когда обобщенный показатель $D_{i}$ был больше 0,37
(0,388). Температура воздуха при этом составляла $-22,4^{\circ} \mathrm{C}$, скорость ветра $1,3 \mathrm{M} / \mathrm{c}$.

Для территории РП 983 (рис. 2) в течение анализируемого периода температура воздуха менялась от $-8,9$ до $-35,4^{\circ} \mathrm{C}$, средняя скорость ветра от 0 до 5 м/с. Число дней, когда величина обобщенного показателя была не меньше 0,37 , составило 6 из 20 дней (9-14 ноября), причем, только в течение 5 дней можно было работать в дневные часы.

Для территории РП 985 (рис. 3) в течение анализируемого периода температура воздуха менялась от $-15,0$ до $-40,1^{\circ} \mathrm{C}$, средняя скорость ветра от 0 до 5 м/с. Число дней, когда величина обобщенного показателя была не меньше 0,37 , составило 6 из 20 дней, причем, только в течение 4 дней можно было работать в дневные часы: 11 и 21 ноября условие возможности работы на открытом воздухе без обморожений выполнялось после 18 часов местного времени.

\section{ЗАКЛЮЧЕНИЕ}

С использованием мультипликативной модели биоклиматического показателя жесткости погоды по фактическим ежедневным данным рассчитана величина обобщенного показателя $D_{i}$ для отдельных территорий РП. Определены комбинации па- 
Использование мультипликативной модели биоклиматического показателя для определения локальных условий безопасности работ на открытом воздухе

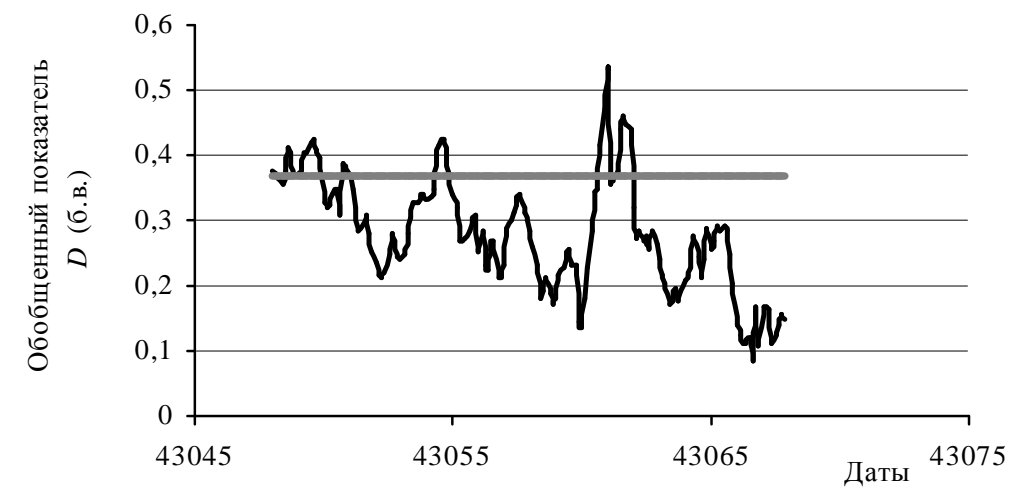

Puc. 3. Изменение обобщенного показателя $D_{i}$ за период 9-28.11.2017 для территории РП 985. Серая линия соответствует выбранному критическому значению обобщенного показателя $D=0,37$

раметров температуры воздуха $\left(t_{i}\right)$ и скорости ветра $\left(v_{i}\right)$, для которых выполняется условие $D_{i} \geq 0,37$, когда сохраняется возможность работы на открытом воздухе без обморожений открытых участков тела. Полученные результаты позволяют установить конкретные интервалы времени работы на открытом воздухе в суровых условиях без риска получения травм от холода. Кроме того могут быть использованы в оперативной работе при планировании работ в суровых погодных условиях, а также для краткосрочного прогноза условий работы на открытом воздухе.

\section{СПИСОК ЛИТЕРАТУРЫ}

1. Двуреченский А. О районе падения частей ракет, которые запустят с Восточного / А. Двуреченский. URL: http://www.roscosmos.ru (дата обращения: 17.10.2017).

2. МР 2.2.7.2129-06.2.2.7. Физиология труда и эргономика: Режимы труда и отдыха работающих в холодное время на открытой территории или в неотапливаемых помещениях : методические рекомендации / Р. Ф. Афанасьева [и др.]. - Введ. 01.11.2006. - 12 с.

3. Прикладная статистика. Основы эконометрики / ред. С. А. Айвазян. - Москва : ЮНИТИ-ДАНА, 2001. Т. 2 : Основы эконометрики. -432 с.

4. Руководство по специализированному климатологическому обслуживанию экономики / под ред. Н. В. Кобышевой. - Санкт-Петербург : Гидрометеоиздат, 2005. - 336 c.

5. Хайруллин К. Ш. Биоклиматические ресурсы России / К. Ш. Хайруллин, В. Н. Карпенко // Энциклопедия климатических ресурсов Российской Федерации. - Санкт-Петербург, 2005. - С. 25-46.

6. Шипко Ю. В. Обобщенный биоклиматический показатель безопасности работ на открытом воздухе в суровых погодных условиях / Ю. В. Шипко, Е. В. Шувакин, А. В. Иванов // Вестник Воронежского государственного университета. Сер. География. Геоэкология. - 2015. - № 3. - C.33-39.
7. Groen G. Wind chill equivalent temperature (WCET). Climatology and scenarios for Schiphol Airport. KNMI / G. Groen. - 2009. - URL: http://www.knmi.nl/samenw/kbs/ doc/WCET-Schiphol.pdf (дата обращения: 17.10.2017).

8. Mohan M. A Modified Approach to Analyze Thermal Comfort Classification / M. Mohan, A. Gupta, S. Bhati // Atmospheric and Climate Sciences. - 2014. - Vol. 4. P. 7-19.

9. Report on wind chill temperature and extreme heat indices: evaluation and improvement projects. - 2003. URL: http:// www.ofcm.gov/jagti/r19-ti-plan/pdf/ entire_r19_ti.pdf (дата обращения: 17.10.2017).

10. Siple P. A. Measurements of dry atmospheric cooling in sub-freezing temperatures / P. A. Siple, C. F. Passel // Proceedings of the American Philosophical Society. - 1945. - Vol. 89. - P. 177-199.

\section{REFERENCES}

1. Dvurechenskiy A. O rayone padeniya chastey raket, kotorye zapustyat s Vostochnogo / A. Dvurechenskiy. URL: http://www.roscosmos.ru (data obrashcheniya: 17.10.2017).

2. MR 2.2.7.2129-06.2.2.7. Fiziologiya truda i ergonomika: Rezhimy truda i otdykha rabotayushchikh v kholodnoe vremya na otkrytoy territorii ili v neotaplivaemykh pomeshcheniyakh : metodicheskie rekomendatsii / R. F. Afanas'eva [i dr.]. - Vved. 01.11.2006. - $12 \mathrm{~s}$.

3. Prikladnaya statistika. Osnovy ekonometriki / red. S. A. Ayvazyan. - Moskva : YuNITI-DANA, 2001. - T. 2 : Osnovy ekonometriki. - $432 \mathrm{~s}$.

4. Rukovodstvo po spetsializirovannomu klimatologicheskomu obsluzhivaniyu ekonomiki / pod red. N. V. Kobyshevoy. - Sankt-Peterburg : Gidrometeoizdat, 2005. $336 \mathrm{~s}$.

5. Khayrullin K. Sh. Bioklimaticheskie resursy Rossii / K. Sh. Khayrullin, V. N. Karpenko // Entsiklopediya klimaticheskikh resursov Rossiyskoy Federatsii. - Sankt-Peterburg, 2005. - S. 25-46.

6. Shipko Yu. V. Obobshchennyy bioklimaticheskiy pokazatel' bezopasnosti rabot na otkrytom vozdukhe $\mathrm{v}$ surovykh pogodnykh usloviyakh / Yu. V. Shipko, E. V. Shu- 
vakin, A. V. Ivanov // Vestnik Voronezhskogo gosudarstvennogo universiteta. Ser. Geografiya. Geoekologiya. 2015. - № 3. - S.33-39.

7. Groen G. Wind chill equivalent temperature (WCET). Climatology and scenarios for Schiphol Airport. KNMI / G. Groen. - 2009. - URL: http://www.knmi.nl/samenw/kbs/ doc/WCET-Schiphol.pdf (data obrashcheniya: 17.10.2017).

8. Mohan M. A Modified Approach to Analyze Thermal Comfort Classification / M. Mohan, A. Gupta, S. Bhati

\section{Суковатов Константин Юрьевич}

кандидат физико-математических наук, научный сотрудник Института водных и экологических проблем Сибирского отделения РАН, доцент Базовой кафедры информационных технологий и физических методов в экологических исследованиях Алтайского государственного университета, г. Барнаул, т. (3852) 666-462, E-mail: skonstantiny@gmail.com

Безуглова Надежда Николаевна кандидат физико-математических наук, старший научный сотрудник Института водных и экологических проблем Сибирского отделения РАН, г. Барнаул, т. (3852) 666-462, E-mail: bezuglovan@ gmail.com
// Atmospheric and Climate Sciences. - 2014. - Vol. 4. P. 7-19.

9. Report on wind chill temperature and extreme heat indices: evaluation and improvement projects. - 2003. URL: http:// www.ofcm.gov/jagti/r19-ti-plan/pdf/ entire_r19_ti.pdf (data obrashcheniya: 17.10.2017).

10. Siple P. A. Measurements of dry atmospheric cooling in sub-freezing temperatures / P. A. Siple, C. F. Passel // Proceedings of the American Philosophical Society. - 1945. - Vol. 89. - P. 177-199.

Sukovatov Konstantin Yurievich

Candidate of Physical and Mathematical Sciences, Researcher of the Institute for Water and Environmental Problems of the Siberian Branch of the Russian Academy of Sciences, Associate Professor of the Base Department of Information Technologies and Physical Methods in Ecological Research at Altai State University, Barnaul, tel. (3852) 666-462, E-mail: skonstantiny@gmail.com

Bezuglova Nadezhda Nickolaevna

Candidate of Physical and Mathematical Sciences, Senior Researcher of the Institute for Water and Environmental Problems of the Siberian Branch of the Russian Academy of Sciences, Barnaul, tel. (3852) 666-462, E-mail: bezuglovan@gmail.com 